\title{
Construcción de micro ciclos en el deporte
}

\author{
Building of \\ micro cycles in sports
}

\author{
EDGARDO ROMERO FRÓMETA (D), MARIO VACA GARCÍA (D), \\ Universidad de las Fuerzas Armadas ESPE, \\ Av. General Rumiñahui s/n y Ambato, Sangolquí, Ecuador \\ *ndjaramillo@espe.edu.ec
}

NARCISA DE JESÚS JARAMILLO ASTUDILLO* (D), MARÍA FERNANDA GRANJA LOOR (D)

\section{RESUMEN}

El presente estudio descriptivo tiene como objetivo el análisis de una estructura intermedia en la planificación del entrenamiento deportivo: el micro ciclo, estructura que tiene su base en los ejercicios que constituyen las sesiones de entrenamiento realizadas durante varios días.

Los resultados muestran la importancia en la construcción de los micro ciclos y cómo influye esta herramienta en la planificación del entrenamiento, favoreciendo al conocimiento de entrenadores y personal relacionado a la actividad física.

Palabras clave: Planificación, micro ciclo, entrenamiento deportivo, ejercicios.

\section{ABSTRACT}

This current descriptive research aims to analyze an intermediate structure in the planning of sports training: the micro cycle, a structure that is based on the exercises, which are made of the training sessions carried out over several days. 
The results show not only the importance in the construction of the micro cycles, but also how this tool influences on the training planning, favoring both the coaches' knowledge and other personnel involved with the physical activity.

Keyword: planning, micro cycle, sports training, exercises.

\section{INTRODUCCIÓN}

\section{Periodización, programa y planificación}

Frecuentemente los términos planificación, programación y periodización se usan como si fueran sinónimos, pero en realidad son diferentes. La planificación es una herramienta que facilita una sólida estructura, organización y contenidos que sirven de guía para el respectivo proceso del atleta, hasta alcanzar sus máximos logros (Camacho, Ochoa, \& Rincón, 2019).

Explican (Rosa \& Saorín, 2014) que los micro ciclos tendrán en cuenta determinar las modalidades de entrenamiento, tanto aquellas que son dominantes y las sesiones son importantes al proporcionar el principal impacto del micro ciclo.

Los autores consideran que el micro ciclo es una unid cualquier deporte que se trate, por la regularidad que establece para los efectos inmediatos, retardados y acumulados de carga y su influencia en la fase de adaptación preconizadas por Selye y Shultz. En consecuencia, las aclaraciones que surjan a respecto del análisis bibliográfico resultan muy útiles para los especialistas.

La periodización contiene la planificación y la programación, la estructura de un plan anual y su contenido (que se compone de medios y métodos de entrenamiento) a medida cambia o se modifica con el tiempo. Por lo tanto, podemos definir la periodización del plan anual como una estructura en el proceso de entrenamiento que divide el año en fases y establece una secuencia de desarrollo de capacidades determinantes y condicionantes (Bompa T. O., 2016), donde el micro ciclo dicta los procedimientos de trabajo.

Para poder ejecutar una planificación se requiere de la periodización, que es un proceso ordenado que permite establecer los entrenamientos, en periodos y objetivos durante un tiempo determinado. Por ende, este proceso debe ser controlado, evaluado y ajustado de manera periódica para que el atleta pueda alcanzar su máximo nivel de rendimiento (Vales, Areces, Arce, \& Torrado, 2017). La periodización en el entrenamiento conlleva a diseñar un plan de entrenamiento con la finalidad de conseguir el máximo rendimiento del deportista, es decir, establecer planificaciones donde se busque el desarrollo integral del deportista y perdure a lo largo de su carrera deportiva. Al hablar de una planificación (Platonov, 2001) la clasificaría en tres instancias: largo plazo con una preparación inicial, de base, específica de base, realización máxima y el mantenimiento de los resultados; a mediano plazo, donde se cuenta con objetivos y directrices que permitan la planificación anual de 2 a 4 años, y por último, a corto plazo, un periodo más reducido y con una 
estructura en temporadas, macro ciclos, meso ciclos, micro ciclos y sesiones de entrenamiento (Henrique, Athayde, Alvarez, \& V, 2019). Estas estructuras son conocidas como ciclos de entrenamiento que constituyen una sucesión lógica donde se modifican las fases en el proceso de entrenamiento y simultáneamente manifiestan la tendencia al desarrollo del entrenamiento, la modificación parcial de la composición de los medios, métodos y el crecimiento de las cargas (León Fuentes, 2011).

Explican (Oruña, I y Peraza,R, 2014) que en la actualidad la dinámica competitiva ha crecido considerablemente para enfrentar las competencias fundamentales, pues hoy en día no tan solo ha crecido el número de competencias, sino que se dispersan por toda la macro estructura del plan. Ello conduce, según (Font, Figueroa, \& Avila, 2019) a evitar la improvisación, a motivar las premisas de una estrategia de trabajo que asegure el desarrollo del entrenamiento, a lograr una continua progresión y mantenimiento de la forma de los deportistas, entre otros.

\section{MÉTODO}

Se recopiló información utilizando motores de búsqueda académicos (Revista Scielo, Dialnet, EfDeportes, Mundo Entrenamiento y Google Académico) relacionados al tema propuesto y bajo selección de criterios. Se ubicaron varios artículos relacionados con el micro ciclo de entrenamiento, que permitieron ayudar a contextualizar, respaldar y realizar el análisis.

\section{Componentes de la planificación deportiva}

El proceso de entrenamiento se estructura en periodos temporales correctamente definidos (Rodríguez, 2010), destacándose la siguiente estructura:

- Macro ciclos: ciclos anuales - preparatorio, precompetitivo, competitivo y transición.

- Meso ciclos: ciclos de 2 a 8 semanas.

- Micro ciclos: ciclos de aproximadamente una semana, conformado por sesión de entrenamiento y unidades de entrenamiento

\section{Micro ciclo}

Se denomina micro ciclo a una serie de sesiones realizadas durante varios días, que comúnmente aseguran la obtención de los objetivos para una etapa dada de la preparación (Garcia, Ruíz, \& Navarro, 1996). La duración de los micro ciclos comprende de 3-4 días hasta 10-14 días, y los más conocidos son los de 6-7 días, que van acorde con la duración de la semana del calendario y, el estilo de vida de los deportistas (García, 2011 ). También existen los micro ciclos del periodo competitivo que se modifican según las necesidades de las futuras competencias (Platonov, 2001). Es probable que el micro ciclo, o programa de entrenamiento semanal sea la herramienta de planificación más importante (Matveev, 1985). Durante el plan anual, la naturaleza y dinámica del micro ciclo cambia de acuerdo con la fase del entrenamiento, los objetivos del entrenamiento y las exigencias fisiológicas y psicológicas afrontadas por el atleta (Bompa, T, 1995). 


\section{Tipos de micro ciclos}

La organización de la carga en cada uno de los micro ciclos varía, y depende en gran medida del objetivo que se persigue (Bompa \& Buzzichelli, 2015). Para secuenciar los distintos micro ciclos se atienden a distintos condicionantes, asociados con el momento de la temporada, el tipo de meso ciclo, el nivel de rendimiento del deportista y la etapa del plan anual por la cual transita el deportista, los deportes de un corto período competitivo son aquellos que necesitan un mínimo de tiempo (Sotelo, 2018). Existen diferentes tipos de micro ciclos dependiendo del objetivo mismo: ajuste, carga, impacto, activación, competición y recuperación (Navarro, 2000).

- Micro ciclo de ajuste: llamado también introductorio o entrante, se diseña para preparar al deportista al siguiente período de entrenamiento o meso ciclo. Este tipo de micro ciclos se ubican al inicio de toda temporada, especialmente porque le permite al entrenador observar el comportamiento del deportista respecto a las cargas planificadas, y hacer cada vez más específicos los contenidos de la planificación.

- Micro ciclo de carga: También recibe el nombre de micro ciclo corriente u ordinario, se caracteriza por presentar un aumento uniforme de la carga, por su volumen considerable y aumento moderado de la intensidad. Este micro ciclo se utiliza con mayor frecuencia en los meso ciclos de preparación general y especial o de acumulación (Figura 1).

\section{Figura 1}

Ejemplo de un microciclo desarrollador en por cientos

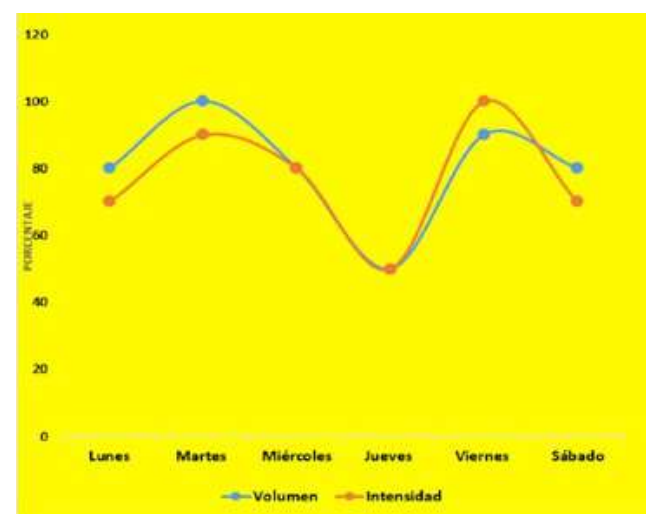

Nota. Este micro ciclo se utiliza con mayor frecuencia en los meso ciclos de preparación general y especial o de acumulación

Observe que en este tipo de micro ciclo el día de mayor carga es el martes y el de mayor intensidad el viernes. El deportista en el micro ciclo precedente descansó el domingo; el lunes realizó una carga media. Comúnmente luego de un domingo de descanso, el lunes el deportista viene con pereza para entrenar y una carga media le permite "despertarse" del descanso y prepararse para recibir la mayor carga el martes. Luego de una carga relativamente baja el jueves, para recuperarse de la carga de los tres días precedentes, con un descanso activo, le sucede la segunda mayor carga de la semana, el viernes y el sábado utiliza una carga media-alta, pues su organismo ha recibido cinco días de carga de diferentes volúmenes e intensidad, lo que conduce a que el sábado esté muy cansado, como consecuencia del fenómeno de huellas. Al bajar la carga el sábado, respecto al viernes, realmente no descendió, debido a sus efectos retardado y acumulado. A continuación, un ejemplo de las unidades de un micro ciclo desarrollador en el alto nivel competitivo. 
Tabla 1

Ejemplo de unidades de un micro ciclo de carga de alto nivel en etapa de preparación general

\begin{tabular}{cc} 
DIRECCIÓN & NO. DE UNIDADES \\
\hline Coordinación técnico-táctica & 4 \\
\hline Rapidez & 3 \\
\hline Fuerza Rápida & 6 \\
\hline Potencia aláctica & 2 \\
\hline Capacidad aláctica & 2 \\
\hline Potencia láctica & 2 \\
\hline Capacidad Láctica & 1 \\
\hline Potencia Aerobia & 2 \\
\hline Capacidad Aerobia & 3 \\
\hline Regeneración & 5 \\
\hline Flexibilidad & 5 \\
\hline Juego & 2 \\
\hline Total & 37
\end{tabular}

Tabla 2

Ejemplo didáctico de la forma de distribuir las unidades en el micro ciclo. Alto Nivel Competitivo, horario de la mañana

\begin{tabular}{c|c}
\hline Lunes & $\begin{array}{c}\text { Flexibilidad, rapidez, coordinación } \\
\text { técnica-táctica, fuerza rápida. }\end{array}$ \\
\hline Martes & $\begin{array}{c}\text { Velocidad, coordinación técnica-táctica, } \\
\text { fuerza rápida }\end{array}$ \\
\hline Miércoles & $\begin{array}{c}\text { Flexibilidad, fuerza rápida, potencia } \\
\text { aláctica. }\end{array}$ \\
\hline Jueves & $\begin{array}{c}\text { Juego, potencia aerobia, capacidad } \\
\text { aerobia. }\end{array}$ \\
\hline Viernes & $\begin{array}{c}\text { Flexibilidad, rapidez, coordinación } \\
\text { técnico-táctica, fuerza rápida. } \\
\text { Sábado }\end{array}$ \\
\hline Coordinación técnica-táctica, fuerza \\
rápida, capacidad láctica, regeneración.
\end{tabular}

Tabla 3

Ejemplo didáctico de la forma de distribuir las unidades en el micro ciclo. Alto Nivel Competitivo, horario de la tarde

\begin{tabular}{c|c}
\hline Lunes & $\begin{array}{c}\text { Potencia } \\
\text { aláctica }\end{array}$ \\
\hline Martes & $\begin{array}{r}\text { Flexibilidad, fuerza rápida, capacidad } \\
\text { aláctica, regeneración }\end{array}$ \\
\hline Miércoles & $\begin{array}{c}\text { Potencia láctica, } \\
\text { capacidad aerobia }\end{array}$ \\
\hline Jueves & $\begin{array}{c}\text { Fuerza rápida, flexibilidad, } \\
\text { regeneración }\end{array}$ \\
\hline Viernes & $\begin{array}{c}\text { Potencia láctica, } \\
\text { capacidad aerobia }\end{array}$ \\
\hline Sábado & $\begin{array}{c}\text { Potencia aerobia, } \\
\text { juego, regeneración }\end{array}$ \\
\hline
\end{tabular}

Nota. Ejemplo de las unidades de un micro ciclo desarrollador en el alto nivel competitivo.

La distribución de las unidades del micro ciclo responde al imperativo del efecto retardado de la carga, todo ello asociado con el fenómeno de huellas. Luego de una carga de fuerza rápida máxima o resistencia, el organismo queda agotado, así que no puede sucederle una carga de rapidez o técnica, que son las primeras que deben aparecer en el orden de una sesión de entrenamiento. En ese mismo orden, una sesión con altas cargas metabólica no debe ser sucedida por una carga de rapidez al siguiente día. Todo ello se asocia con el empleo de sesiones de regeneración y el fenómeno de huellas. 


\section{Tabla 4}

Ejemplo de unidades de un micro ciclo de carga de en etapa de iniciación deportiva

\begin{tabular}{cc} 
DIRECCIÓN & NO. DE UNIDADES \\
\hline Coordinación/Técnica-táctica & 5 \\
\hline Rapidez & 4 \\
\hline Fuerza rápida & 2 \\
\hline Potencia Aerobia & 1 \\
\hline Capacidad Aerobia & 3 \\
\hline Regeneración & 3 \\
\hline Flexibilidad & 2 \\
\hline Acondicionamiento & 2 \\
\hline Juego & 3 \\
\hline Total & 37
\end{tabular}

Tabla 5

Ejemplo didáctico de la forma de distribuir las unidades en un micro ciclo de iniciación deportiva en etapa de preparación especial

\begin{tabular}{c|c}
\hline Lunes & $\begin{array}{c}\text { Flexibilidad, rapidez, coordinación } \\
\text { técnico-táctica }\end{array}$ \\
\hline Martes & $\begin{array}{c}\text { Rapidez, coordinación técnico-táctica, fuerza } \\
\text { rápida, acondicionamiento juego, regeneración }\end{array}$ \\
\hline Miércoles & $\begin{array}{r}\text { Rapidez, coordinación técnico-táctica, } \\
\text { flexibilidad, potencia aerobia }\end{array}$ \\
\hline Jueves & $\begin{array}{r}\text { Acondicionamiento, juego, capacidad aerobia, } \\
\text { regeneración }\end{array}$ \\
\hline Viernes & $\begin{array}{r}\text { Rapidez, coordinación técnico-táctica, fuerza } \\
\text { rápida, capacidad aerobia. }\end{array}$ \\
\hline
\end{tabular}

Nota. La distribución de las unidades del micro ciclo responde al imperativo del efecto retardado de la carga, todo ello asociado con el fenómeno de huellas.
Al diseñar las sesiones diarias en el micro ciclo, es muy común observar que el día lunes la rapidez precede a la coordinación y a la preparación técnico-táctica, y esta, a la fuerza rápida. No puede ser de otra manera. La rapidez es una capacidad motora que tiene como premisas que el atleta no esté cansado y, por otra parte, para la realización de las unidades técnico-táctica se requiere también un estado en que el deportista no experimente fatiga, pues se pueden producir interferencias en el aprendizaje. Asimismo, como en el horario de la mañana se realizó un entrenamiento dirigido al desarrollo y los mecanismos de producción y energía anaerobio, como es el caso de la rapidez y la coordinación, pues en un período de 4-6 horas ya están recuperados y, en consecuencia, el organismo del deportista está presto a realizar un trabajo de potencia anaerobia aláctica.

Este trabajo del lunes no crea interferencias fisiológicas para continuar el trabajo de rapidez al día siguiente luego de un descanso reparador y sistematizar el desarrollo del día precedente, pero se introduce en la tarde una unidad de flexibilidad y luego se desarrolla la dirección anaerobia aláctica, como continuación del trabajo realizado la tarde anterior, pero esta vez dirigido a la capacidad aláctica.

Considerando que el día martes es el más adecuado para la mayor carga, por la densidad relativamente baja del lunes, ya que ese día el atleta llegó al entrenamiento luego de un día de descanso, el domingo, y generalmente no tiene deseos de entrenar. Explica (Guillamon, A y Avilés, G, 2014) que normalmente los micro ciclos finalizarán con una jornada de descanso. Por lo tanto, la primera sesión del siguiente micro ciclo no puede ser importante, pues el descanso reduce la predisposición del deportista hacia las 
cargas altas. Es lógico que el martes concluya con cargas bajas de regeneración y facilitar así la recuperación para el trabajo del día siguiente en la mañana, que está dirigido a la flexibilidad, la fuerza rápida y la potencia aláctica, para concluir en la tarde con unidades consecutivas de potencia láctica y capacidad aerobia, según lo exige el orden del desarrollo biológico de esas direcciones en lo que ya se había explicado sobre la articulación vertical y horizontal de la carga, es decir, la adaptación cronológica.

La sesión del miércoles se realiza sobre la base de las huellas del lunes y el martes, en consecuencia, es un trabajo más ligero que el martes, pero más fuerte que el lunes. Como el martes en la tarde hubo regeneración, que pudo haberse realizado con sesiones de masaje, hidromasaje, trote lento, masaje de hielo, etc., el miércoles se desarrolla nuevamente a primera hora la flexibilidad, la fuerza rápida y la potencia, que es un trabajo con carreras cortas a alta rapidez en series, dirigida a la resistencia anaerobia.

Así, siguiendo el orden lógico, en la tarde se extiende el trabajo a la potencia láctica y culmina con un trabajo en la dirección aerobia.

Ya a este nivel, el atleta tiene sobre sí las huellas de 18 unidades de entrenamiento, realizadas en 6 sesiones, así que el día jueves se debe realizar una inflexión de la carga, con un descanso relativo. Así es que ese día lo inicia con potencia aerobia y su extensión, la capacidad aerobia, pero de bajo volumen para que, en la tarde, se introduzca una sesión de flexibilidad, seguramente con estiramientos, seguida de juego y regeneración, para provocar con ese cambio de actividad una recuperación más rápida sobre el fundamento de la inhibición por inducción negativa, propia del descanso activo.
El día viernes en la mañana se realiza un trabajo parecido al lunes y martes en igual momento, pues luego del descanso activo de la tarde el organismo se encuentra con una predisposición efectiva para realizar la segunda mayor carga de la semana. Es así que en la tarde se dirige el entrenamiento hacia la potencia láctica y la capacidad aerobia.

A esta altura del micro ciclo el atleta comienza a experimentar el cansancio de la semana, propio de las huellas dejadas sobre él por las cargas precedentes. Entonces, el día sábado se inicia el trabajo con preparación técnico táctica, dirigido al perfeccionamiento de esas acciones estando el atleta en un estado de cansancio, tal como ocurre en las competencias y se continúa todo el trabajo del día desarrollando direcciones asociadas con la resistencia. En este caso se desarrollan en la mañana en ese orden: la capacidad láctica y la regeneración, para que en la tarde se concluya con una sesión dirigida a la potencia aerobia y al juego, finalizando la parte principal de la sesión con actividades de regeneración.

Si se analiza esa distribución de unidades y de sesiones, se podrá notar que en ellas se ha valorado la forma en que las unidades deben articular vertical y horizontalmente sobre la base de la consideración de los fundamentos fisiológicos que le dan lugar a la sucesión del tipo de carga que se aplique.

Resulta relevante que es solo un ejemplo de las incalculables formas en que las unidades pueden ser articuladas, y que no ha tenido en cuenta a ningún deporte en específico, ya que se trata de la metodología de planificación del micro ciclo como un recurso didáctico, pero que permite fundamentar la lógica del pensamiento del entrenador cuando dosifica la carga, tanto en el micro ciclo como en las sesiones de entrenamiento. 
- Micro ciclo de choque: Su característica radica en que el volumen y la intensidad se elevan y tienden a igualarse, buscan generar nuevos procesos de adaptación en el organismo. Al ser la intensidad y el volumen altos, se genera una fatiga profunda en el deportista, este tipo de micro ciclo suele utilizarse en los meso ciclos básicos desarrolladores (Navarro, 2020).

Este tipo de micro ciclo genera un impacto grande sobre la influencia biológica de la carga y es común utilizarlo cuando el deportista demuestra en el test un estancamiento de la evolución del rendimiento (Figura 2).

\section{Figura 2}

Ejemplo de un micro ciclo de choque en por ciento

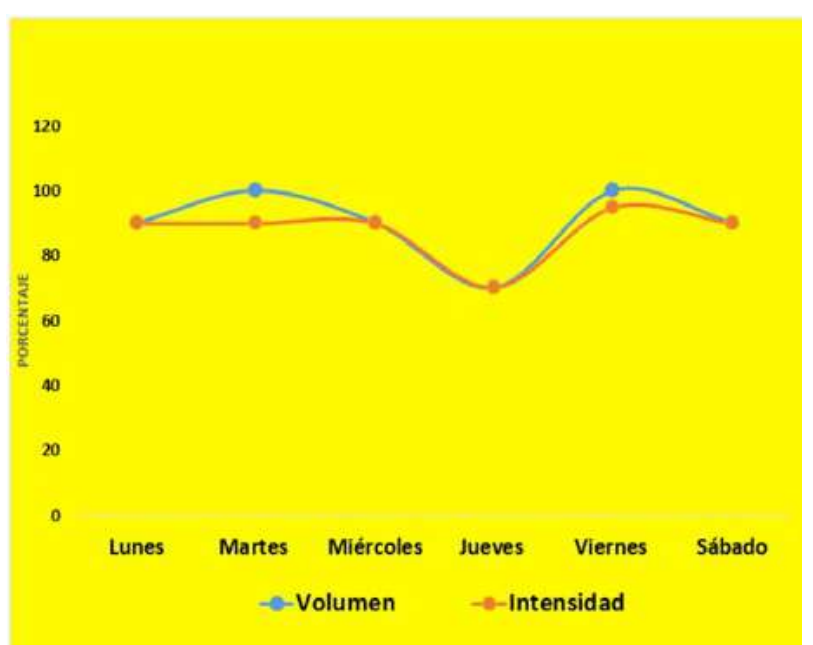

Nota. Este tipo de micro ciclo genera un impacto grande sobre la influencia biológica de la carga y es común utilizarlo cuando el deportista demuestra en el test un estancamiento de la evolución del rendimiento.
- Micro ciclo de activación: también denominado de aproximación, acercamiento o precompetitivo, se califica por utilizar cargas específicas muy similares a las de competición. El objetivo es el de preparar al deportista para las condiciones de la competición.

Hay que ada ptar al organismo del deportista al mismo régimen de vida a la que se verá sometido en la etapa competitiva. Si en esa etapa va a desayunar a las 7 am, a esa misma hora desayunará todos los días de la semana del micro ciclo de activación o precompetitivo. Asimismo, si la competencia principal será realizada de noche, pues debe entrenar de noche y a la misma hora en que será la competencia, readaptando el régimen del día al sistema que mantendrá en la semana de entrenamiento de la competencia principal. Eso incluye también los horarios de la alimentación y descanso. La figura 3 es solo un ejemplo de ello.

\section{Figura 3}

Ejemplo de micro ciclo de activación o precompetitivo

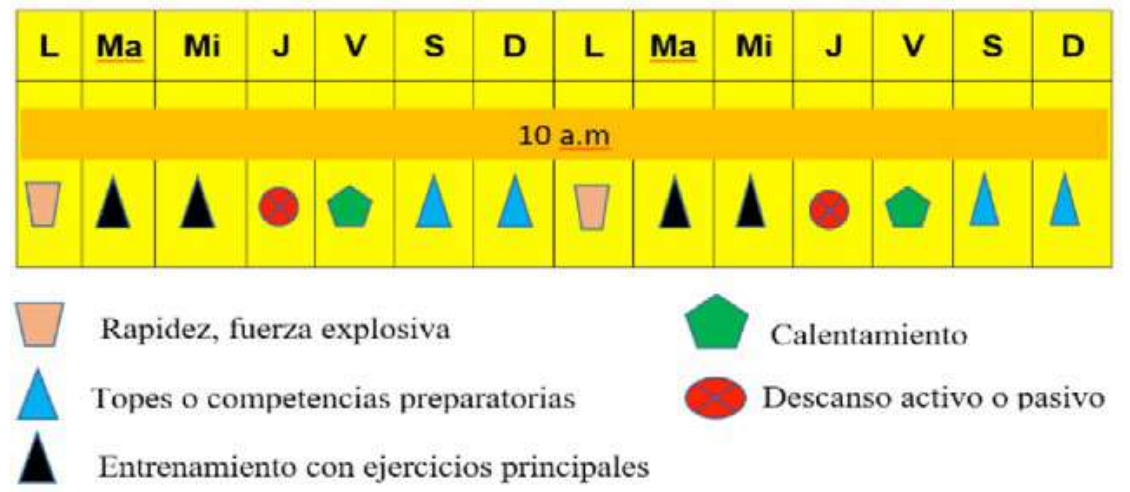

Nota. Hay que adaptar al organismo del deportista al mismo régimen de vida a la que se verá sometido en la etapa competitiva. 
- Micro ciclo competitivo: se caracteriza por integrar en su organización las competencias importantes, sesiones suplementarias y procedimientos de recuperación.

Una vez aplicados varios micro ciclos de aproximación o de activación, se obtendrá el micro ciclo semanal de mayor relieve para enfrenar la competencia principal. En los micro ciclos de activación se prueban diversas rutinas, hasta lograr la rutina semanal de mayor relieve para el deportista y es la que se adopta en el micro ciclo competitivo.

- Micro ciclo de recuperación: Este tipo de micro ciclos se realizan después de las competencias principales o de la realización de micro ciclos de impacto, con la finalidad de restablecer el equilibrio del organismo. Sus objetivos principales están enfocados en potenciar los procesos óptimos de recuperación y proporcionar al organismo una mayor posibilidad de adaptación, que se alcanza mediante medios como la hidroterapia, la crioterapia, masajes y otros métodos en sentido general de regeneración. Se puede evitar muchos tipos de lesiones, gracias a la correcta periodización del descanso, obteniendo mejores resultados de inadaptación.

\section{Construcción del micro ciclo}

El intervalo entre dos unidades de entrenamiento debe ser lo suficientemente extenso como para permitir la recuperación necesaria y adecuada para lograr el máximo efecto de entrenamiento de la siguiente unidad.
La recuperación se acelera si se introducen unidades de regeneración en el micro ciclo.

Los micro ciclos bien construidos reducen la monotonía en el entrenamiento, si se los arma correctamente.

Las cargas de altas demandas metabólicas o fuerza máxima deben llevarse a cabo en días de óptima capacidad y nunca luego de días de altas demandas, especialmente si involucra al sistema de energía anaerobio-láctico.

En la tabla siguiente se establece el orden preferencial para el desarrollo de diferentes unidades en el micro ciclo, según la lista que permite autoevaluar si se sigue el orden adecuado y más abajo en las unidades del micro ciclo, las posibles unidades que pueden ser desarrolladas en un micro ciclo dado. Como es lógico, resulta solo un ejemplo, pues la vivencia de cada entrenador es mucho más rica, pero ofrece una idea de la lista de unidades que hay que conformar para elaborar cada micro ciclo.

Existe un orden, producto de las investigaciones realizadas, que, aunque no es dogmático, orienta en casa sesión de entrenamiento consecutivo en que deben desarrollarse las diferentes direcciones del rendimiento:

1. Rapidez- coordinación técnico-táctica o técnica.

2. Fuerza explosiva-fuerza rápida, fuerza máxima

3. Potencia aláctica capacidad aláctica

4. Potencia láctica, capacidad láctica.

5. Resistencia de fuerza

6. Acondicionamiento

7. Potencia aerobia, capacidad aerobia 
Para elaborar un micro ciclo es preciso tener en cuenta: en qué período o fase se encuentran, pues la carga, el número de unidades y su tipo va a depender de la tendencia que sigue el entrenamiento y, sobre todo, de las direcciones del rendimiento que están siendo desarrolladas en forma más acusada. Así, en plena preparación general, serán utilizadas un número mayor de unidades dirigidas a las direcciones condicionantes del rendimiento, tales como la capacidad aerobia, la resistencia de la fuerza, la flexibilidad y otras, mientras que en etapa o fase de preparación especial primarán aquellas unidades dirigidas al desarrollo de las direcciones determinantes del rendimiento, tales como la capacidad y la potencia anaerobia láctica y aláctica, la rapidez, la fuerza rápida, la dirección técnico-táctica y otras. Ello, como es lógico, facilitará identificar las unidades a cubrir y su cantidad, en correspondencia con los objetivos del micro ciclo que se trate.

Combinación de unidades en la parte principal de la sesión de entrenamiento.

En el proceso de entrenamiento las transferencias positiva, negativa y neutra están siempre actuando sobre el atleta. Corresponde al entrenador regular ese efecto, pero siempre pensando que esas contradicciones subsisten en una unidad que genera saltos positivos en el desarrollo del deportista.

Para los entrenadores es muy importante conocer la sucesión de las diferentes unidades, sobre todo aquellas que desarrollan preferentemente la rapidez, la fuerza, la resistencia y la influencia de estos ejercicios sobre la efectividad de la enseñanza de la técnica en el conjunto de ejercicios del deporte dado y considerando el grado de transferencia de los hábitos somáticos vegetativos. Todo ello asociado con el efecto Sechenov del descanso activo, el cual consiste en el restablecimiento de la capacidad de trabajo es más eficaz cuando no se trata de un descanso pasivo, sino de una actividad contrastante.

La clase más efectiva resulta aquella donde se solucionan las tareas del desarrollo de una serie de direcciones del rendimiento, incluyendo la enseñanza y perfeccionamiento del nivel de la técnica. Este sistema de las clases fue muy utilizado en Cuba por el ruso Leonid Chervakov, en la formación del triplista y se emplea actualmente de forma amplia en todo el deporte cubano. Indica (Romero, 2006) que en la elaboración de un micro ciclo deben cumplirse determinadas reglas y destaca entre ellas considerar que al emplearse diversas demandas en el propio micro ciclo, no sería necesario emplear una recuperación completa.

Siguiendo esa línea, hay que tener en cuenta que la efectividad de los ejercicios de velocidad-fuerza realizados después de los ejercicios de velocidad, alcanza resultados favorables. Esos resultados aconsejan, entonces, desarrollar los ejercicios de fuerza-rápida luego de los ejercicios de rapidez.

La efectividad de los ejercicios de velocidad-fuerza realizados en la clase después de la carga de fuerza disminuye su efectividad. Al final de los 20 minutos de descanso la capacidad para la actividad de velocidad-fuerza se recupera. Estos resultados demuestran que si el entrenador pretende utilizar ejercicios de fuerza-rápida después de ejercicios propiamente de fuerza, debe hacer una pausa de no menos de 20 minutos al concluir los ejercicios de fuerza. 
La efectividad de los ejercicios de velocidad-fuerza, ejecutados después del trabajo de resistencia, disminuye profundamente. En los siguientes 20 minutos de descanso llevan al organismo a la pérdida de la capacidad para la actividad de velocidadfuerza.

El aprendizaje y perfeccionamiento de la técnica debe realizarse al principio de la parte principal de la clase, después del calentamiento o posterior a los ejercicios que desarrollan preferentemente la rapidez, pero con un volumen que no lleve al atleta al agotamiento.

Los ejercicios que desarrollan la resistencia deben realizarse después de los ejercicios que desarrollan marcadamente la rapidez y la fuerza. El volumen de los ejercicios de carácter de fuerza y rapidez permite el desarrollo de la resistencia, y la recuperación de la capacidad de trabajo de los atletas se produce más rápido después de los ejercicios de rapidez y fuerza.

La mejor efectividad de la enseñanza de la técnica y del desarrollo de las capacidades motoras en una clase se logra cuando los ejercicios se emplean en la parte principal siguiendo la siguiente sucesión:

Ejercicios que desarrollan preferentemente la rapidez, ejercicios que se utilizan con el fin de desarrollar la técnica, ejercicios que desarrollan la fuerza y ejercicios que desarrollan la resistencia. Durante la inclusión en la clase de tales tipos de ejercicios se debe mantener la misma sucesión.
La distribución señalada para los ejercicios, de acuerdo con su tendencia, no se debe adoptar dogmáticamente. En algunos casos se puede adoptar otra sucesión dependiendo de la etapa o período de entrenamiento o los objetivos de la clase. Se trata de que se entienda que el orden indicado es el ideal.

En la parte principal de la clase de entrenamiento intervienen múltiples unidades que algunos llaman acentos, que al ser combinados óptimamente contribuyen a una efectiva transferencia de hábitos. El ejemplo que a continuación se muestra fue utilizado con gran éxito en el entrenamiento en las edades infantiles del atletismo cubano, y como tal, constituye sólo una aproximación a la organización de la parte principal de las clases de entrenamiento, en consideración a su estructura metodológica.

\section{RESULTADOS}

El micro ciclo es una estructura pequeña, lo componen una serie de sesiones realizadas durante varios días que aseguran la consecución conjunta de los objetivos para una etapa dada de la preparación. La duración del micro ciclo comprende de 3 a 4 días hasta 10 a 14 días. Los micro ciclos más utilizados son los de 7 días (6). El micro ciclo toma diferentes nombres de acuerdo a la calidad y cantidad de estímulos que conformen el contenido del mismo. Esta estructura tiene como premisa la ejecución de las actividades propias de la preparación que requieren de repetición permanente, deben tener en cuenta para su planificación las siguientes normas: 
- Precisa correlación entre el volumen e intensidad en la aplicación de las cargas, observando su principio ondulatorio.

- Debe prestar atención a la ejecución reiterada de un conjunto de habilidades donde se relacionen cambios de orientación.

- Es muy importante formular los objetivos que lógicamente se han derivado del meso ciclo correspondiente.

- La planificación y desglose de las tareas o actividades que se complementan o deben complementarse en el entorno del micro ciclos responde al logro de los objetivos propuestos.

- Su planificación requiere especial atención del entrenador, quien debe analizar las cargas que aplica (volumen intensidad) y el tiempo de recuperación entre la unidad de entrenamiento y la siguiente, o entre sesiones de entrenamiento.

\section{Impacto en el micro ciclo}

- Representa los primeros fragmentos respectivamente acabados del proceso de entrenamiento.

- Su estructura y contenido determinan la calidad del proceso de entrenamiento.

- Es probablemente la herramienta más importante de la planificación.

- Permite organizar en mejores condiciones el proceso de entrenamiento.

- Posee alta adaptabilidad a las circunstancias del momento: entrenamiento actual, disposición al trabajo, condiciones climáticas, control carga, etc...

- Aseguran la consecución conjunta de los objetivos para una etapa dada de la preparación.
- Comprenden una serie de sesiones donde se aplica un tipo específico de trabajo antes de cambiar los objetivos del entrenamiento o descanso.

\section{Beneficios de los micro ciclos:}

- Previene la práctica de reiterar los mismos ejercicios todos los días, con lo cual no se realizarán entrenamientos monótonos ni aburridos, obteniendo mejores resultados, además se varían los tipos de entrenamiento y cargas, motivando al deportista a realizar cada uno de ellos con una mejor predisposición.

- Perfecciona lentamente y sin estancamiento alguno la programación que se tiene para el entrenamiento a realizar.

- Permite ahorrar, esfuerzo y tiempo para alcanzar los objetivos previstos con una mejor eficacia y eficiencia.

- Se puede evitar muchos tipos de lesiones por entrenamiento gracias a la correcta periodización de los descansos, obteniendo mejores resultados y garantizando un mejor desempeño.

\section{CONCLUSIONES}

1. Los tipos de micro ciclo a utilizarse dependen del nivel del deportista y de la etapa de entrenamiento anual en que se encuentra.

2. Los micros ciclos desarrolladores y de choque son típicos de la preparación general y especial, mientras que aquellos de activación, precompetitivos o competitivos responden a la etapa competitiva. 
3. Las unidades de entrenamiento de todo el micro ciclo, como de las sesiones de entrenamiento, guardan un orden preferencial, están asociadas con el tipo de carga y su influencia en el metabolismo, por tanto, las unidades de rapidez y técnica preceden a las restantes y las anaerobias a las aerobias.

4. La estructura de los micro ciclos variará en función de la elección del deporte, el nivel o/ y experiencia deportiva, la edad y la etapa del entrenamiento por la cual se encuentra el deportista, tanto a largo plazo como en el plan anual.

5. Un micro ciclo bien formado debe garantizar el efecto Sechenov del descanso activo y con él la inhibición por inducción negativa, en consecuencia, las cargas deben ser muy variadas.

\section{REFERENCIAS}

Benítez, J. A. (2014). Vitónica. https://www.vitónica.com/ musculación/organización-del-entrenamiento-macrociclomicrociclo-y-mesociclo-iii

Bompa, T. (1995). Teoría y metodología del entrenamiento deportivo. Kendall and Hunt.

Bompa, T. О. (2016). Periodización del entrenamiento deportivo (Cuarta ed.). PaidoTribo.

Bompa, T., \& Buzzichelli, C. (2015). Periodization Training for Sports (Tercera ed.). Estados Unidos: Human Kinetics. http://www.humankinetics.com/products/all-products/ periodization-training-for-sports-3rd-edition-ebook
Camacho , J., Ochoa, N., \& Rincón, N. (2019). REVISIÓN TEÓRICA DE LA PLANIFICACIÓN TRADICIONAL Y CONTEMPORÁNEA EN EL ENTRENAMIENTO DEPORTIVO. Actividad Física y Deporte, 171-181.

Font, J., Figueroa, M., \& Avila, R. (2019). Una variante de microciclo para la preparación y el control del rendimiento físico. Dilemas Contemporáneos: Educación, Política y Valores.

García, J., Ruíz, J., \& Navarro, M. (1996). Planificación del Entrenamiento Deportivo. Gymnos.

García, O. (2011). Aplicación práctica para la planificación del entrenamiento en la modalidad deportiva del lanzamiento del tejo. (T. Guterman, Ed.) Lecturas Educación Física y Deportes, 16(163). Recuperado el 19 de Septiembre de 2020, de https://www.efdeportes.com/efd163/ entrenamiento-del-lanzamiento-del-tejo.htm

Guillamon, A y Avilés,G. (Enero de 2014). Propuesta práctica de planificación del entrenamiento para un nadador de 200 metros libres. (T. Guterman, Ed.) Lecturas Educación Física y Deportes., 18(188). Recuperado el 19 de Septiembre de 2020, de https://www.efdeportes.com/efd188/ planificacion-del-entrenamiento-nadador-de-200-metros. $\mathrm{htm}$

Guterman, T. (Ed.). (Septiembre de 2010). La planificación Deportiva y sus Componentes. Lecturas Educación Física y Deportes, 15(148). Recuperado el 19 de Septiembre de 2020, de https://www.efdeportes.com/efd148/laplanificación-deportiva-y-sus-componentes.htm 
Henrique, P., Athayde, A., Alvarez, D., \& V, S. (2019). Clasificación de Conceptos Relacionados con el Entrenamiento Deportivo. Escuela Abierta.

León Fuentes, C. E. (2011). Repositorio ESPE. hHtp://repositorio. espe.edu.ec/bitstream/21000/5190/1/T-ESPE-033090. pdf

Matveev, L. P. (1985). Fundamentos del entrenamiento deportivo. Editorial Raduga.

Navarro , F. (2000). Apuntes del master de alto rendimiento deportivo. COE Universidad Autónoma.

Oruña, I y Peraza,R. (Febrero de 2014). Guía para la planificación del entrenamiento deportivo. (T. Guterman, Ed.) Lecturas Educación Física y Deportes, año 18(189). Recuperado el 19 de Septiembre de 2020, de https://www. efdeportes.com/efd 189/guia-para-la-planificación-delentrenamiento.htm

Platonov. (2001). Teoría General del Entrenamiento Deportivo Olímpico. Paidotribo.
Rodríguez, J. M. (2010). La planificación deportiva y sus componentes. EFDeportes, 148.

Romero, E. (2006). El entrenamiento Deportivo, Conceptos y metodología. (UCCFD, Ed., \& E. Romero, Recopilador) Habana, Habana, Cuba. Recuperado el 25 de Noviembre de 2020

Rosa, A., \& Saorín, G. (Enero de 2014). Propuesta práctica de planificación del entrenamiento para un nadador de 200 metros libres. (T. Guterman, Ed.) Lecturas Educación Física y Deportes, 18(188). Recuperado el 25 de Noviembre de 2020, de https://www.efdeportes.com/efd188/ planificación-del-entrenamiento-nadador-de-200-metros. $\mathrm{htm}$

Sotelo, I. (2018). El Microciclo de Entrenamiento. Mundo Entrenamiento.

Vales, A., Areces, A., Arce, C., \& Torrado, J. (2017). La planificación del entrenamiento deportivo: cambios vinculads a las nuevas formas de entender las estructuras deportivas contemporáneas. Revista Da Faculdade Educacao Física de UNICAMP. 


\section{BIOGRAFÍA DE LOS AUTORES}

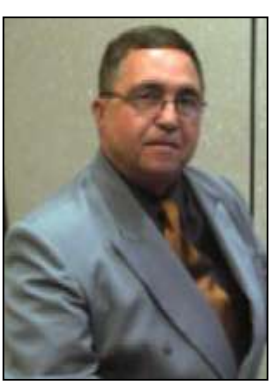

EDGARDO ROMERO F.

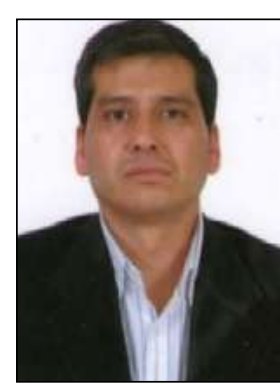

MARIO VACA GARCÍA
Ph. D. en Cultura Física, es también licenciado en Ciencias de la Cultura Física, especialidad de Atletismo. En su carrera profesional ha trabajado en la Universidad de Ciencia de la Cultura Física y el Deporte "Manuel Fajardo" en La Habana, Cuba.

Actualmente es profesor titular y coordinador de investigaciones del Departamento de Ciencias Humanas y Sociales de la Universidad de las Fuerzas Armadas ESPE, titular jubilado de la UCCFD de La Habana, Cuba, docente de Maestría en entrenamiento Deportivo de la UFA-ESPE, Universidad Central del Ecuador y Universidad Estatal de Milagro.
Máster en Docencia Universitaria, graduado en la Escuela Politécnica del Ejército (Ecuador), es también Licenciado en Ciencias de la Educación en la especialización de Educación Física de la Universidad Central del Ecuador. En su carrera profesional ha trabajado como entrenador de natación de la selección de Manabí, entrenador de la selección de triatlón de la Policía Nacional, metodólogo técnico en la Concentración Deportiva de Pichincha y Ministerio del Deporte, docente en la Universidad de las Fuerzas Armadas ESPE, Universidad Técnica del Norte, Universidad Católica de Cuenca, Universidad Central del Ecuador y Universidad Técnica de Ambato.

Actualmente, se desempeña como director (e) de la Carrera de Pedagogía de la Actividad Física Deportes y Recreación en la UFA-ESPE.

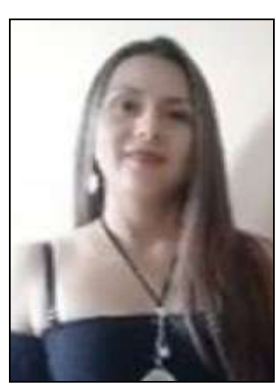

NARCISA JARAMILLO A.

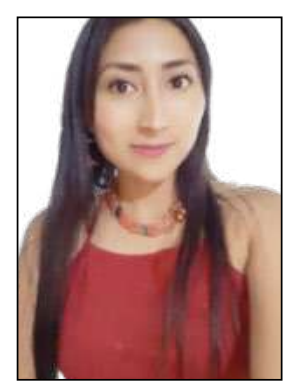

MARIA FERNANDA GRANDA L.

Tecnóloga en Administración Turística graduada en la Escuela Politécnica del Ejército (Ecuador). Ha participado en los programas de vinculación con la sociedad de la Universidad de Fuerzas Armadas ESPE en la Fundación Children International y en instituciones educativas de las parroquias de Conocoto, Sangolqui y Amaguaña. Actualmente se encuentra realizando su trabajo de titulación de la Carrera de Licenciatura en Ciencias de la Actividad Física, Deportes y Recreación en la Universidad de las Fuerzas Armadas ESPE.
Estudió en el Colegio Técnico Humanístico "Quito" donde obtuvo el título de Bachiller en Ciencias. Ha participado en talleres recreativos en "Casa de las religiosas de Jesús y María" y es entrenadora de futbol en la Academia "Rey de Cancha". Actualmente se encuentra realizando su trabajo de titulación de la Carrera de Licenciatura en Ciencias de la Actividad Física, Deportes y Recreación en la Universidad de las Fuerzas Armadas ESPE. 